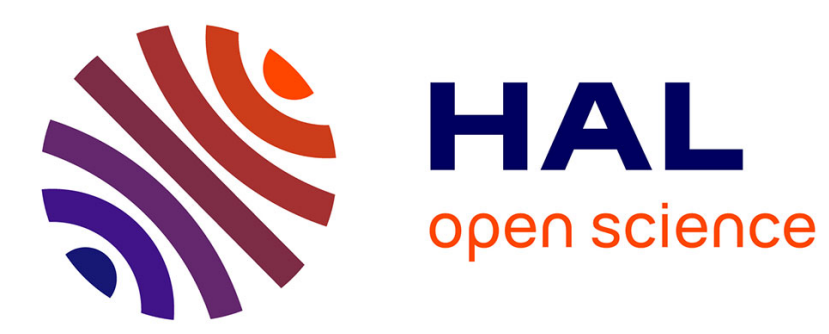

\title{
Active chromatic depth from defocus for industrial inspection
}

Benjamin Buat, Pauline Trouvé-Peloux, Frédéric Champagnat, Guy Le Besnerais, Thierry Simon

\section{- To cite this version:}

Benjamin Buat, Pauline Trouvé-Peloux, Frédéric Champagnat, Guy Le Besnerais, Thierry Simon. Active chromatic depth from defocus for industrial inspection. SPIE Photonics Europe 2020, Apr 2020, Strasbourg, France. hal-03523311

\section{HAL Id: hal-03523311 \\ https://hal.science/hal-03523311}

Submitted on 12 Jan 2022

HAL is a multi-disciplinary open access archive for the deposit and dissemination of scientific research documents, whether they are published or not. The documents may come from teaching and research institutions in France or abroad, or from public or private research centers.
L'archive ouverte pluridisciplinaire HAL, est destinée au dépôt et à la diffusion de documents scientifiques de niveau recherche, publiés ou non, émanant des établissements d'enseignement et de recherche français ou étrangers, des laboratoires publics ou privés. 


\title{
Active chromatic depth from defocus for industrial inspection
}

\author{
Benjamin Buat ${ }^{\mathrm{a}}$, Pauline Trouvé-Peloux ${ }^{\mathrm{a}}$, Frédéric Champagnat ${ }^{\mathrm{a}}$, Guy Le Besnerais ${ }^{\mathrm{a}}$, and \\ Thierry Simon ${ }^{\mathrm{b}}$ \\ ${ }^{a}$ DTIS, ONERA, Université Paris Saclay F-91123 Palaiseau - France \\ bUUT Figeac, Nayrac 46100 Figeac - France
}

\begin{abstract}
In this paper we propose a new concept for a compact 3D sensor dedicated to industrial inspection, combining chromatic Depth From Defocus (DFD) and structured illumination. Depth is estimated from a single image using local estimation of the defocus blur. As industrial objects usually show poor texture information, which is crucial for DFD, we rely on structured illumination. In contrast with state of the art approaches for active DFD, which project sparse patterns on the scene, our method exploits a dense textured pattern and provides dense depth maps of the scene. Besides, to overcome depth ambiguity and dead zone of DFD with a classical camera, we use an unconventional lens with chromatic aberration, providing spectrally varying defocus blur in the camera color channels. We provide comparisons of depth estimation performance for several projected patterns at various scales based on simulation and real experiments. The proposed method is then qualitatively evaluated on a real industrial object. Finally we discuss the perspectives of this work especially in terms of co-design of an 3D active sensor using DFD.
\end{abstract}

Keywords: active 3D, depth from defocus, surface inspection, chromatic aberration

\section{INTRODUCTION}

Surface inspection is a major issue in industrial manufacturing in sectors such as aeronautic and automotive. It can be carried out during the production phase of the equipment, particularly during the machining of metal parts, in order to check if the equipment fits the specification, but also during the control phases after a given time of use. In both contexts, 3D sensors have to be cheap, compact and to be usable at few centimeters from the surface of the object to provide a large-scale 3D profile with a sub-millimeter precision.

Estimation of 3D surface of an inspected object is usually carried out thanks to data collected by tactile sensors (e.g., probing). While the inspection is extremely precise (submicrometric) with such approaches, the measurement is very localized and the operation turns out to be time consuming and cumbersome. Contact-free methods are also used, mostly based on optical sensors. ${ }^{1}$ Unlike tactile sensors, optical ones have now a limited cost, are compact and allow non-destructive, large scale and fast inspection of the object, thus are very promising for surface inspection.

A conventional approach to optical 3D estimation is stereoscopy, which is based on parallax between images from different cameras. However, this method requires at least two cameras, increasing the system dimension and requiring cameras relative calibration and synchronization. Most recently, other 3D measurement techniques using only one camera have been developed, such as methods exploiting defocus blur for depth estimation. There are two main approaches exploiting defocus, which are Depth From Defocus ${ }^{2}$ (DFD) and Depth From Focus ${ }^{3}$ (DFF). DFF uses a large number of images captured while changing the optical settings and depth is estimated by the search of the best focused image in the image stack. Processing is simple and fast, but the main limitation of the methods is in the recording of the image stack which requires that the scene and the camera position be fixed during certain amount of time. On the other hand, DFD requires only one or two images and depth is estimated by measuring local defocus blur. Acquisition is then easier and provide a larger field of applications than DFF, but the processing is more difficult, specially in the Single Image case (SIDFD) as both scene and

Further author information: (Send correspondence to Benjamin Buat)

Benjamin Buat: E-mail: benjamin.buat@onera.fr, Telephone: +33 786762729 


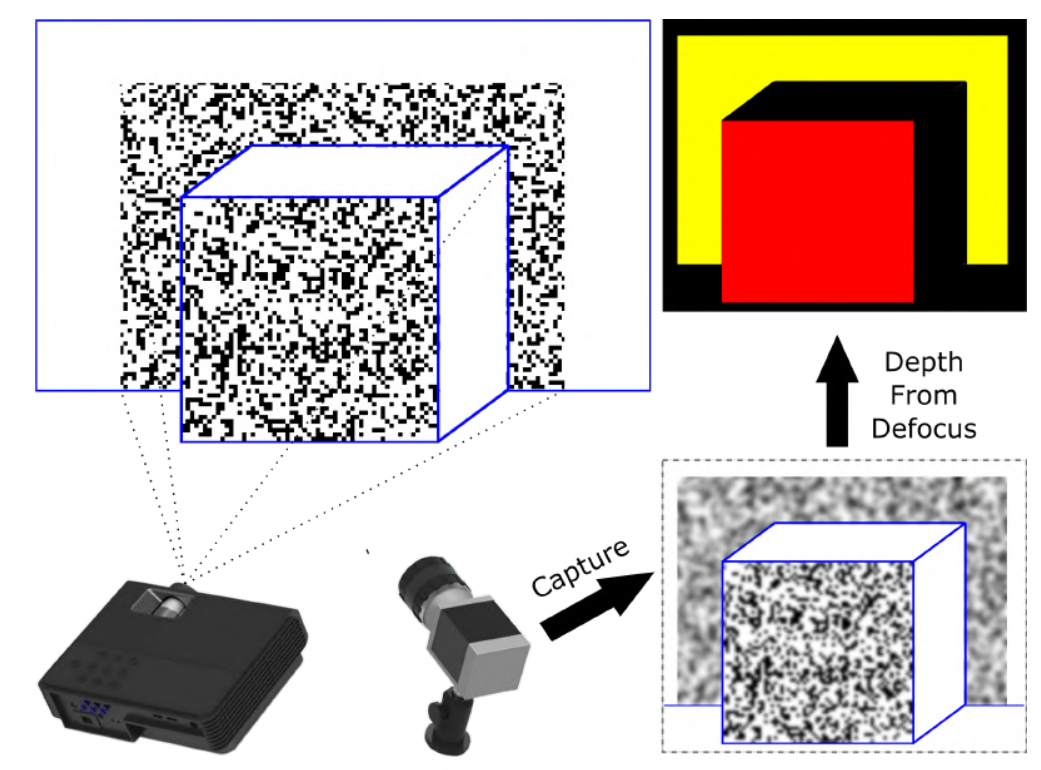

Figure 1. Principle of the proposed active DFD system. A dense textured pattern is projected onto the scene, a camera equipped with a chromatic lens record an image, the DFD algorithm uses the variations of blur over the camera color channels to determine a dense depth map.

blur are unknown. Besides, SIDFD suffers from ambiguity (the same blur amount can be measured in front or behind the in-focus plane) and from a "dead zone" where no measurement is possible in the camera depth of field. Unconventional optics dedicated to DFD have been proposed, based on coded aperture, ${ }^{4}$ chromatic aperture ${ }^{5}$ or chromatic lens, ${ }^{6}$ improving depth estimation performance. Finally, plenoptic cameras have been developed, ${ }^{7}$ composed of microlenses array placed in front of the detector to generate multi-view images. Requiring a single image, these approaches lead to compact 3D devices.

However, passive 3D estimation techniques are much less effective when the objects inspected lack texture and are poorly illuminated. To overcome these limitations, active approaches can be thought of such as laser triangulation, interferometry, Time-of-Flight measurement or structured illumination. ${ }^{1}$ Laser triangulation is an easy method to put in place but is rather slow due to the point by point (or line by line) depth estimation. Interferometry is based on the measure of interference fringe created by the superimposition of two light beams split from an initial beam. One of the divided beams is reflected on a test surface, the other one on a reference surface, implying a difference of optical path length between them, and producing an interference when they are combined. This is the most accurate 3D measurement method but it is more efficient at small depth range (millimeter). Time-of-Flight sensors which measure the round trip time of a light signal to calculate the distance between the camera and an object, are rather used to estimate the depth of a large scale scene over several meters than within few tens of centimeters of the sensors. Finally, structured illumination relies on the projection of a known pattern onto a scene and the calculation of depth information thanks to the deformation of the pattern on different surfaces. Structured illumination seems the most suited to industrial inspection. However, using such method involves several limitations: the depth estimation performance depends on the baseline between the camera and the projector, reducing the compactness of the system and entailing missing data during the correspondence process due to occlusion, shadows or reflections. Moreover, camera and projector must be calibrated in relation to each other and both must be perfectly in focus, these two steps are not easily performed and can lead to errors during the measurement.

In order to overcome the aforementioned limitations, we propose to conduct industrial surface inspection using a combination of a single image Depth From Defocus approach and a structured illumination. A projector is used to add an artificial texture onto the object and hence improve defocus blur estimation. As depth estimation performance is not related to the distance between projector and camera, a compact system can still be obtained 
without occlusion. We also use an unconventional chromatic camera dedicated to DFD to obtain large depth estimation range without ambiguity nor dead zone. The open issue of the proposed method then lies in the choice of a projected pattern dedicated to Depth From Defocus. We propose here comparisons of several patterns in terms of depth estimation performances, based on simulated images and using the same DFD algorithm. We also study the influence of the pattern scale on the performance. We show that a random noise pattern gives the best performance with the proposed DFD algorithm, at a scale where the smallest structural element corresponds to half the size of the processed patch.

\subsection{Related works}

Active DFD or DFF Active depth estimation methods using defocus blur have been proposed in the literature using either DFF ${ }^{8-11}$ or SIDFD. ${ }^{12-15}$ In this paper, we focus on SIDFD, which is more simple to conduct experimentally since it does not require to dynamically control optical parameters during acquisition. Note that most of the active DFD and DFF methods, except, ${ }^{8,9}$ use the defocus blur from the projector.

Choice of projected pattern The main question in active DFD or DFF is the choice of the projected pattern. Most of methods use evenly spaced vertical lines ${ }^{9,11,12,14}$ or evenly spaced dots. ${ }^{10,13,15}$ Blur is then estimated by measuring the spread or the brightness of each elements of the pattern in the image. While being accurate, these methods only produce sparse depth map with depth information only at the position of each dots/lines, which can not be too close to avoid overlapping. In the context of color video processing, Moreno et al. ${ }^{13}$ estimate a dense depth map by merging sparse depth measurements with a color segmentation of the image, an approach which is not possible with grayscale inspection images. Nayar et al. ${ }^{8}$ developed a method using a dense illumination pattern in the form of a black and white grid with optimized grid parameters to improve depth estimation performance, however in a classical two-frames DFD framework.

Avoiding depth ambiguity of SIDFD To avoid depth ambiguity with respect to the in-focus plane, it is customary in SIDFD to exploit the blur on one side of the infocus plane, ${ }^{13,15}$ thus reducing the depth range. Another approach involves the use of several projection patterns focused at different depths in a single snapshot. For example, Masuyama et al. ${ }^{14}$ develop a projection system consisting of multiple color pattern placed at different distances in front of a light source, each pattern being focused at a different position in the scene. This approach however limits the number of depths that can be estimated at the number of pattern used, in such case only five. Girod et al. ${ }^{12}$ use anisotropic aperture or astigmatic optics for the projector to obtain anisotropic defocus blur whose orientation varies in front or behind the in-focus plane.

\subsection{Contributions and paper organization}

In most of the aforementioned papers the blur is generated by a projector having a small depth of field. Besides, the projected pattern is usually sparse and so is the depth information. In contrast, we propose to exploit the defocus blur of the camera while working within the projector depth of field. This allows the use of unconventional camera dedicated to DFD such as a chromatic lens as in ${ }^{6}$ and thus avoid ambiguity and dead zone of DFD, which increases the depth estimation range. Moreover, in opposition to previous works, we propose to project a dense texture on the scene and to use a patch based DFD algorithm which provides us with dense depth maps although with a spatial resolution related to the size of the patch. This questions the choice of an optimal texture for DFD performance. In this paper we compare in simulations the performance of several potential patterns at various scales to select the one that performs the best with the proposed DFD algorithm. Finally we present the first experimental results of the proposed active chromatic DFD approach on an industrial object.

The paper is organized as follow: In Section 2, we present the principle of the active chromatic DFD system. Section 3 describes the chromatic DFD algorithm used for depth estimation. Then we evaluate and compare the depth estimation performances of different projected patterns by simulation in Section 5 . We validate experimentally our system quantitatively and qualitatively in Section 6. Conclusion remarks and discussions about the perspectives of this work are given in Section 7. 


\section{ACTIVE CHROMATIC DEPTH FROM DEFOCUS}

Figure 1 presents a schematic view of the complete Active Chromatic DFD system. It is made-up of a projector and of a camera equipped with an unconventional lens having chromatic aberration. A dense sharp texture is projected onto the scene and estimated blurs over the three color channels of the recorded image are used to determine depth with a SIDFD algorithm. In the following, we first recall the principle of DFD and then discuss about the benefit brought by chromatic aberration in the system.

\subsection{Depth From Defocus principle}

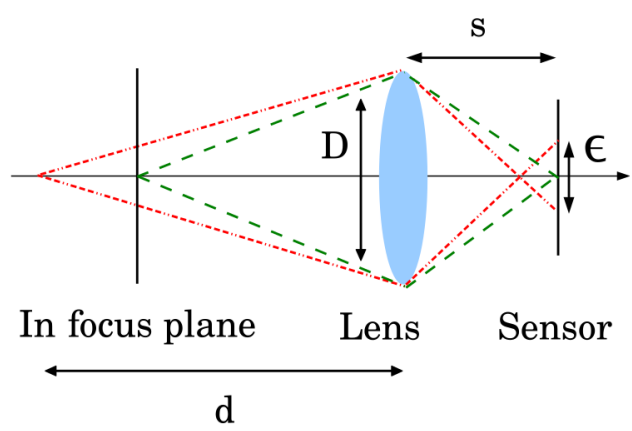

Figure 2. Schematic view of the DFD principle considering an optical system with a conventional lens. In green, the object is in the focus plane, in red it is defocused.

Depth From Defocus is a passive depth estimation method based on the relation between defocus blur and depth. ${ }^{2}$ As illustrated in Figure 2, if an object point source is situated before or after the plan of in-focus of an imaging system, its image - referred to as the Point Spread Function (PSF) - shows defocus blur. Using the thin lens law the size of the defocus blur $\epsilon$ can be determined by equation (1):

$$
\epsilon=D s\left|\frac{1}{f}-\frac{1}{d}-\frac{1}{s}\right|
$$

where $D$ is the lens diameter, $f$ is the focal length, $d$ is the distance from point source to the lens and $s$ is the distance from the sensor to the lens. Various models have been proposed to approximate the defocus blur function but in practice a two-dimensional Gaussian model $^{2}$ has been largely employed. The parameter of interest of the Gaussian model is the standard deviation which specifies the blur level in the defocus image. Here we propose to use a standard deviation given by $\sigma=\rho \epsilon$ where $\rho$ is a factor empirically fixed at 0.3 .

\subsection{Chromatic Depth From Defocus}

The main disadvantage of SIDFD is the depth ambiguity. Indeed an object placed in front of the plane of in-focus and an other object placed behind it can be seen with the same blur amount. As in Trouvé et al, ${ }^{6}$ we propose to use a lens with chromatic aberrations instead of a traditional lens. Longitudinal chromatic aberration leads to a spectral variation of the in-focus plane. Thus an object captured by a RGB color camera coupled to a chromatic lens produces R,G,B channel images with different blurs. Blur from each channel R,G and B are uncorrelated and for each depth there is only one triplet of PSFs (see Figure 4). The depth ambiguity problem is then removed. Another advantage of this chromatic sensor is that dead zone - depth zones close to the in-focus plane where blur is not measurable - is no longer an issue. Indeed with chromatic aberration, for each depth at least one channel is blurred. Thus the addition of the chromatic lens improves accuracy of the depth estimation over a larger range. 


\subsection{Active Chromatic DFD}

Chromatic DFD, like traditional DFD and other passive depth estimation methods is extremely sensitive to scene texture. If an object has an homogeneous surface, DFD algorithms are not capable to measure any defocus blur and thus no depth information can be estimated. In our field of application of industrial surface inspection, most objects are metallic parts and are almost textureless. To deal with such objects, we propose to project dense pattern to create an artificial texture used by the DFD algorithm to estimate depth. A dense pattern is projected onto the scene and remains in-focus in the whole range of depth. A snapshot consisting of 3 images $\mathrm{R}, \mathrm{G}, \mathrm{B}$ is taken with the sensor. The SIDFD algorithm described in the Section 3 is used to estimate depth from the scene.

\section{SIDFD ALGORITHM}

In this paper, we use the SIDFD algorithm proposed, in the context of passive chromatic DFD, by Trouvé et al. ${ }^{6}$ This algorithm is based on a Bayesian formalism of the PSF estimation problem using scene and image prior. Depth is estimated among a set of potential depths using a selection criterion derived from a maximum likelihood approach, calculated on image patches. In the following, we briefly explain the main principles of this algorithm.

\subsection{Image model}

Defocus blur is a spatially varying blur, so an image patch is usually modeled with the local convolution of a scene patch with the PSF and addition of random acquisition noise. Using the vector representation on image and scene patches we have:

$$
\mathbf{Y}=H(z) \mathbf{X}+\mathbf{N}
$$

with $\mathbf{Y}=\left[\mathbf{y}_{R}^{t} \mathbf{y}_{G}^{t} \mathbf{y}_{B}^{t}\right]^{t}, \mathbf{X}=\left[\mathbf{x}_{R}^{t} \mathbf{x}_{G}^{t} \mathbf{x}_{B}^{t}\right]^{t}$, where for each channel $c, \mathbf{y}_{c}$ (respectively $\mathbf{x}_{c}$ ) collects $N$ pixels of the image (resp. scene) patch in the lexicographical order. $\mathbf{N}$ stands for the noise process which is modeled as a zero mean white Gaussian noise (WGN) with variance $\sigma_{n}^{2}$. Moreover the matrix $H(z)$ writes:

$$
H(z)=\left[\begin{array}{ccc}
H_{R}(z) & 0 & 0 \\
0 & H_{G}(z) & 0 \\
0 & 0 & H_{B}(z)
\end{array}\right] .
$$

Each $H_{c}(z)$ is a convolution matrix which depends on the defocus PSF of the channel $c . N$ is the length of each vectors $\mathbf{y}_{c}, M$ the length of each vector $\mathbf{x}_{c}$ thus each $H_{c}$ is a convolution matrix of size $N \times M$. Note that the proposed formalism allows to model both 3CCD and color filter array (CFA) sensors. Modeling a CFA sensor just amounts to remove adequate lines from full convolution matrices $H_{c}$.

\subsection{Scene model}

In the context of local PSF estimation, a Gaussian prior on the scene is often very effective as shown for instance in $^{16}$. However when dealing with color data the components in the RGB decomposition are partially correlated. Following Condat ${ }^{17}$ we propose to use the luminance $(\mathrm{L})$ and the red-green $\left(\mathrm{C}_{1}\right)$ and blue-yellow chrominance $\left(\mathrm{C}_{2}\right)$ decomposition instead of the RGB decomposition using the transform:

$$
\left[\begin{array}{c}
\mathbf{x}_{R} \\
\mathbf{x}_{G} \\
\mathbf{x}_{B}
\end{array}\right]=T \otimes \mathbf{I}_{M, M}\left[\begin{array}{c}
\mathbf{x}_{L} \\
\mathbf{x}_{C_{1}} \\
\mathbf{x}_{C_{2}}
\end{array}\right]
$$

where $\otimes$ stands for the Kronecker product, $\mathbf{I}_{M, M}$ is the identity matrix of size $M \times M$ and:

$$
T=\left[\begin{array}{ccc}
\frac{1}{\sqrt{3}} & \frac{-1}{\sqrt{2}} & \frac{-1}{\sqrt{6}} \\
\frac{1}{\sqrt{3}} & \frac{1}{\sqrt{2}} & \frac{-1}{\sqrt{6}} \\
\frac{1}{\sqrt{3}} & 0 & \frac{2}{\sqrt{6}}
\end{array}\right]
$$


According to Condat ${ }^{17}$, the three components of the luminance/chrominance (LC) decomposition can be assumed to be uncorrelated. We then use the Gaussian prior:

$$
p\left(\mathbf{X}^{L C} ; \sigma_{x_{C}}^{2}\right) \propto \exp \left(-\frac{\left\|D_{C} \mathbf{X}^{L C}\right\|^{2}}{2 \sigma_{x_{C}}^{2}}\right)
$$

where $X^{L C}=\left[x_{L}^{t} x_{C_{1}}^{t} x_{C_{2}}^{t}\right]^{t}$ and:

$$
D_{C}=\left[\begin{array}{ccc}
\sqrt{\mu_{c}} D & 0 & 0 \\
0 & D & 0 \\
0 & 0 & D
\end{array}\right]
$$

Parameter $\mu_{C}$ is the ratio of the luminance and the chrominance variances. As $\operatorname{in}^{17} \mu_{c}$ is fixed at 0.04 .

\subsection{Likelihood marginalisation}

Using the proposed luminance and chrominance decomposition, the image formation model of equation 2 becomes

$$
\mathbf{Y}=H_{C}(z) \mathbf{X}^{L C}+\mathbf{N}
$$

with

$$
H_{C}(z)=H(z) T \otimes \mathbf{I}_{M, M}
$$

Inference about the model can then be conducted using the likelihood function:

$$
p\left(\mathbf{Y} ; z, \alpha, \sigma_{n}^{2}\right)=\left|\frac{Q_{z}}{2 \pi}\right|_{+}^{\frac{1}{2}} \exp \left(-\frac{1}{2} Y^{t} Q_{z} Y\right),
$$

where

$$
\begin{aligned}
Q_{z} & =\frac{P_{z}}{\sigma_{n}^{2}} \\
P_{z} & =I-H_{C}(z)\left(H_{C}^{t} H_{C}(z)+\alpha D_{C}^{t} D_{C}\right)^{-1} H_{C}(z)^{t} .
\end{aligned}
$$

Parameter $\alpha=\sigma_{n}^{2} / \sigma_{x_{C}}^{2}$ can be interpreted as the inverse of a signal to noise ratio and $\left|Q_{z}\right|_{+}$is the product of the non zero eigenvalues of $Q_{z}$.

\subsection{Depth estimation algorithm}

Depth estimation from RGB images with chromatic aberrations is conducted with the method described in ${ }^{6}$. In order to reduce the number of parameters, the marginal likelihood (10) is maximized with respect to the noise variance $\sigma_{n}^{2}$. This leads to $\widehat{\sigma}_{n}^{2}=\mathbf{Y}^{t} P_{z} \mathbf{Y} /(3 N-3)$. Replacing $\widehat{\sigma}_{n}$ into equation (10) gives:

$$
p\left(\mathbf{Y} ; z, \widehat{\sigma}_{n}^{2}, \alpha\right) \propto\left|P_{z}\right|_{+}^{\frac{1}{2}}\left(\mathbf{Y}^{t} P_{z} \mathbf{Y}\right)^{-\left(\frac{3 N-3}{2}\right)} .
$$

Assuming a finite set of potential PSFs, maximization of this generalized likelihood amounts to the criterion for depth estimation :

$$
\begin{aligned}
\widehat{k}, \widehat{\alpha} & =\arg \min _{k, \alpha} G L\left(z_{k}, \alpha\right) . \\
G L(z, \alpha) & =\left|P_{z}\right|_{+}^{-1 /(3 N-3)} \mathbf{Y}^{t} P_{z} \mathbf{Y} .
\end{aligned}
$$

Parameter $\alpha>0$ fixes the inverse SNR for the considered patch. $k$ is the index of depth within the finite set of potential depth values $\left\{z_{1}, \ldots, z_{k}, . . z_{K}\right\}$. Details on the implementation of the algorithm are given in papers. ${ }^{6,16}$ Potential PSF triplets are calibrated for each depth as described in the next section. 


\section{EXPERIMENTAL SETTINGS}

\subsection{System description}

The sensor used is a UI-1240SE from IDS company with a resolution $1280 \times 1024$ pixels and a pixel size of $3.45 \mu \mathrm{m}$. The unconventional lens with chromatic aberration used during the experimentation described in this paper was originally designed in. ${ }^{6}$ For this lens, the focal length of each color channel is respectively $f_{G}=25 \mathrm{~mm}$, $f_{R}=25.09 \mathrm{~mm}$ and $f_{B}=24.9 \mathrm{~mm}$, and the lens is open at $f / 4$. In the following, we present results using this lens in the range of $310-340 \mathrm{~mm}$, which is typical in the context of industrial inspection. For each color channel, blur is modeled by a Gaussian PSF of standard deviation $\sigma_{c}$ that is calibrated as described in Section 4.2.

The pattern is projected with a commercial home projector Qumi Q8 of resolution $1920 \times 1080$ pixels with a lens focal length of $15.8 \mathrm{~mm}$ and open at $f / 1.7$. This type of projector is typically optimized to be used between 2 and $4 \mathrm{~m}$ but its usage is still possible down to $70 \mathrm{~cm}$ of the scene. Depth estimation being done thanks to camera blur, no additional blur should come from the projector, so the position of the projector is essential in our method. Therefore, the projected pattern must be in-focus on the whole working range of the camera, in other words the depth of field of the projector should be higher than the working range of $310-340 \mathrm{~mm}$. In practice, we place the projector at $80 \mathrm{~cm}$ from the scene, its depth of field being $70 \mathrm{~mm}$ at this distance.

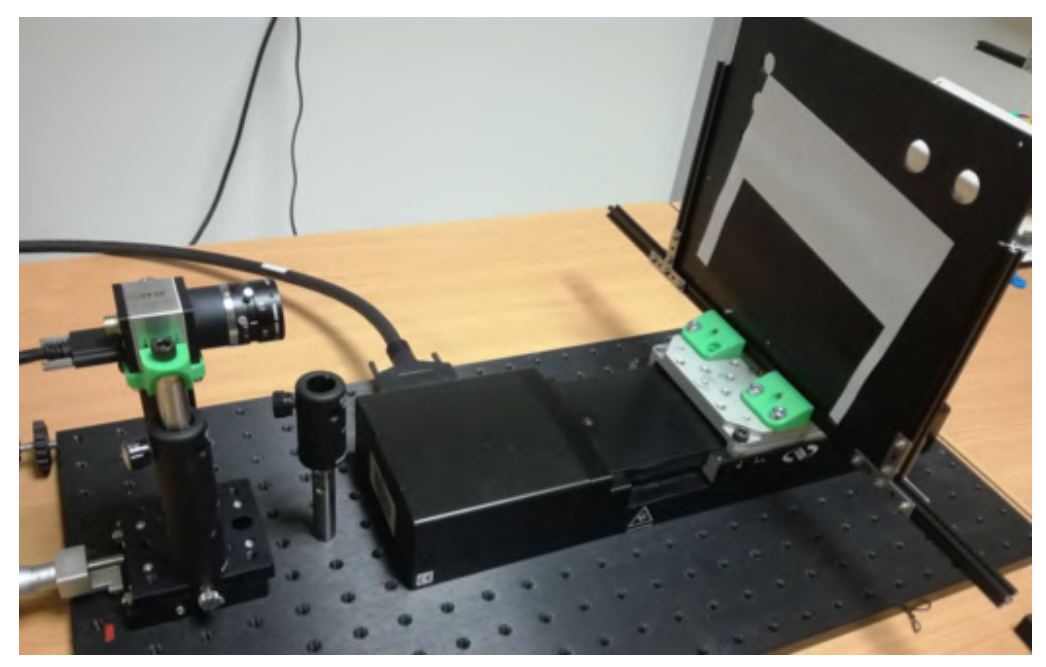

Figure 3. Experimental settings. For calibration a black-and-white edge image printed on a sheet of paper is moved using a motion controller. For each position, the PSF standard deviation is estimated on the image center.

\subsection{System calibration}

As described in Section 3, the proposed DFD algorithm extracts depth from the estimation of PSFs, which is performed by selecting one PSF among a set of potential PSFs using a selection criterion derived from a maximum likelihood approach. Thus, our method requires the calibration of the camera in order to obtain PSFs at each potential distance of the working range. As we choose to simply model the PSF with a Gaussian, we perform calibration with a black-and-white edge image printed on a sheet of paper and pasted on a rigid planar surface as shown in Figure 3. The calibration pattern is moved using an ESP300 motion controller from Newport 3. This controller can be computer programmed and has a micrometric displacement resolution and micrometric precision. PSFs are estimated with a simple knife-edge blur estimation algorithm. ${ }^{18}$ In practice, PSFs are calibrated in the center of the image, with a step of one millimeter between 310 and $340 \mathrm{~mm}$.

\subsection{Focus plane setting}

In our method, depth estimation performance is related to the ability to distinguish the variation of defocus blur with depth. With the proposed setting, as we use a chromatic lens designed in previous work, ${ }^{6}$ the only remaining free parameter is the focus plane position. Thus the choice of the camera focus, which sets blurs variation, is 
decisive. To make this choice, several focus positions for the proposed system have been experimentally compared, two of them are displayed in Figure 4 : in the first one (a), camera focus (of the green channel) is in the middle of the working range $(325 \mathrm{~mm})$. In this case blur of each channel varies slowly and PSF standard deviations are relatively small, i.e., all the channels are nearly focused. Hence, color channel blur variations will be hardly measurable in practice. In the second one (b), camera focus is set further away of the working range, and blur variation is more easily observable. To conclude, the second configuration presented (b) is chosen for our experiment and is used in the following of this paper.

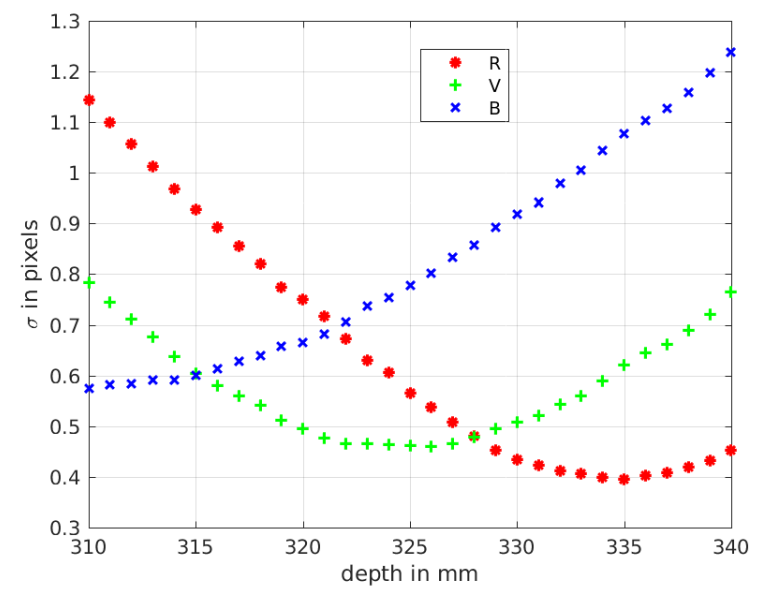

(a)

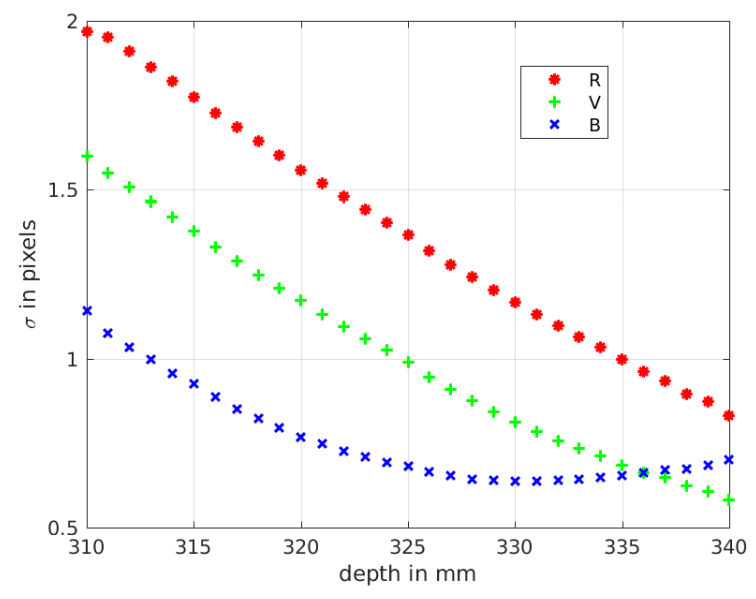

(b)

Figure 4. Experimental variation of PSF standard deviation $\sigma$ with depth. (a) Green channel focused at 325 mm, (b) Green channel focused at $345 \mathrm{~mm}$.

\section{SIMULATIONS}

The main question raised by active DFD is the choice of an optimal pattern to project for the best performance of the algorithm. In this section, we will compare by simulations the performances of several patterns at various scales to select the one that performs the best with the proposed DFD algorithm.

\subsection{Database}

To perform simulations consistent with the industrial inspection context, we build a database of metallic scenes. Hence we select 12 metal sharp scenes from the Salzburg texture image database. ${ }^{19}$ Examples of the proposed scenes are shown in Figure 5.
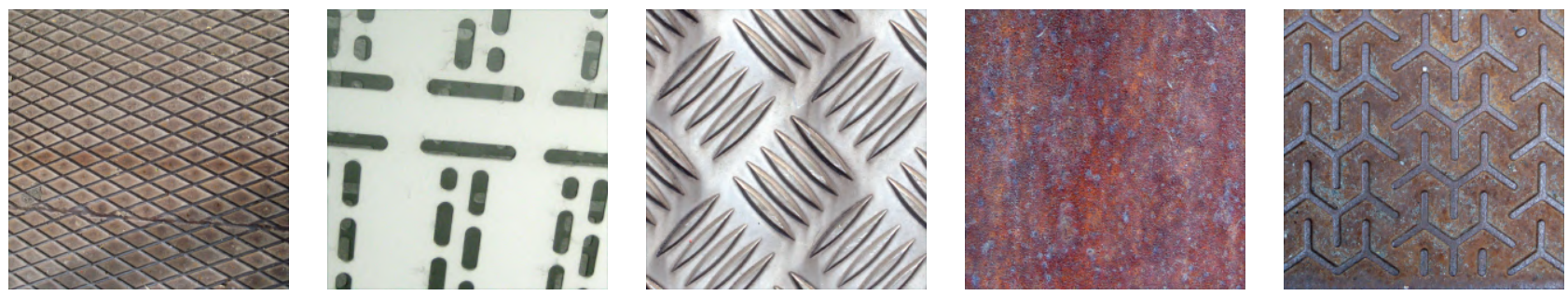

Figure 5. Five of the twelve metal scenes used during the simulation, extracted from the Salzburg texture image database. ${ }^{19}$ 


\subsection{Projected patterns and scale consideration}

In the following, we use the term structural element to define the smallest element of the pattern : this element is a square of $s \times s$ pixels of the same value. Several pattern have been tested and the most relevant ones are presented hereinafter and shown in Figure 6 :

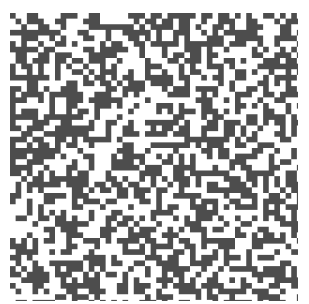

(a)

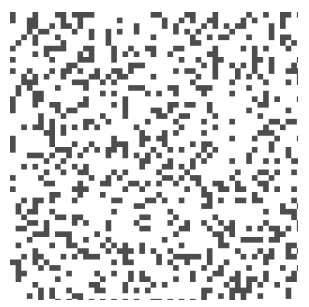

(b)

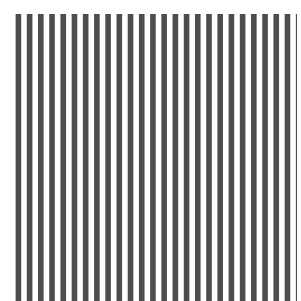

(c)

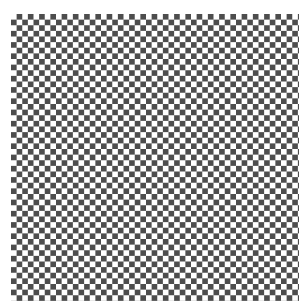

(d)

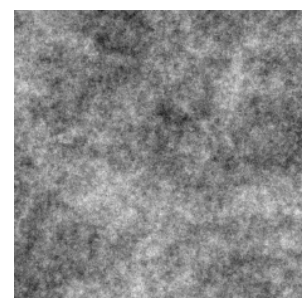

(e)

Figure 6. Illustration of the projected patterns used during the simulation. (a) binary random 50/50, (b) binary random 25/75, (c) vertical lines, (d) checkerboard, (e) Gaussian random.

(a) Binary random pattern 50/50 : each structural element of the pattern has a $50 \%$ chance of being black or white.

(b) Binary random pattern 25/75: each structural element of the pattern has a $25 \%$ chance of being black and $75 \%$ chance of being white.

(c) Line pattern : alternation of white and black vertical lines of structural elements. This is the historical pattern used in active DFD..$^{9,12}$

(d) Checkerboard pattern : alternation of white and black structural elements vertically and horizontally. This is the pattern used by Nayar et al. ${ }^{8}$ for their active DFD method.

(e) Gaussian random pattern : each structural element of the pattern has a value between 0 and 255 while respecting the Gaussian distribution model described in equation (6) in Section 3.2. A Gaussian pattern is then consistent with the scene model of the proposed algorithm.

In the proposed simulation, we also change the scale of the projected pattern (size of the structural element $s$ ), as illustrated in Figure 7 .

\subsection{Evaluation}

In our experiment we settle on patches of size $11 \times 11$ pixels, which is a good compromise between texture information in patch and computational time.

One simulation consists in the following steps : a pattern and size of structural element (from $s=1$ to 10 pixels) is chosen and is applied on one of the twelve metal scenes. One distance from the scene between 310 and $340 \mathrm{~mm}$ is selected and the PSF previously obtained by calibration at this distance is convoluted to the patterned scene to simulate defocus blur. A white gaussian noise of standard deviation of $1 \%$ is then added to this blurred image to simulate realistic camera acquisition. Finally, the DFD algorithm is applied and a depth map is estimated. To evaluate the quality of this estimation, RMSE is calculated between estimated depth and true depth. This processus is repeated for each of the potential patterns, with each of the 10 structural element sizes, at each of the 30 potential distances and for each metal scene.

Note that to simplify the simulation, we did not take into consideration the magnification of the projector. In practice, there is a change of scale of the pattern when the distance to the projector increases but in the proposed simulation, we neglect this effect and the patterns keep the same size regardless of distance.

Depth estimation results are summarized in Figure 8. Average of RMSE for each metal scene is displayed with respect to structural element sizes $s$ for each pattern. To improve visualization of the results, the average 


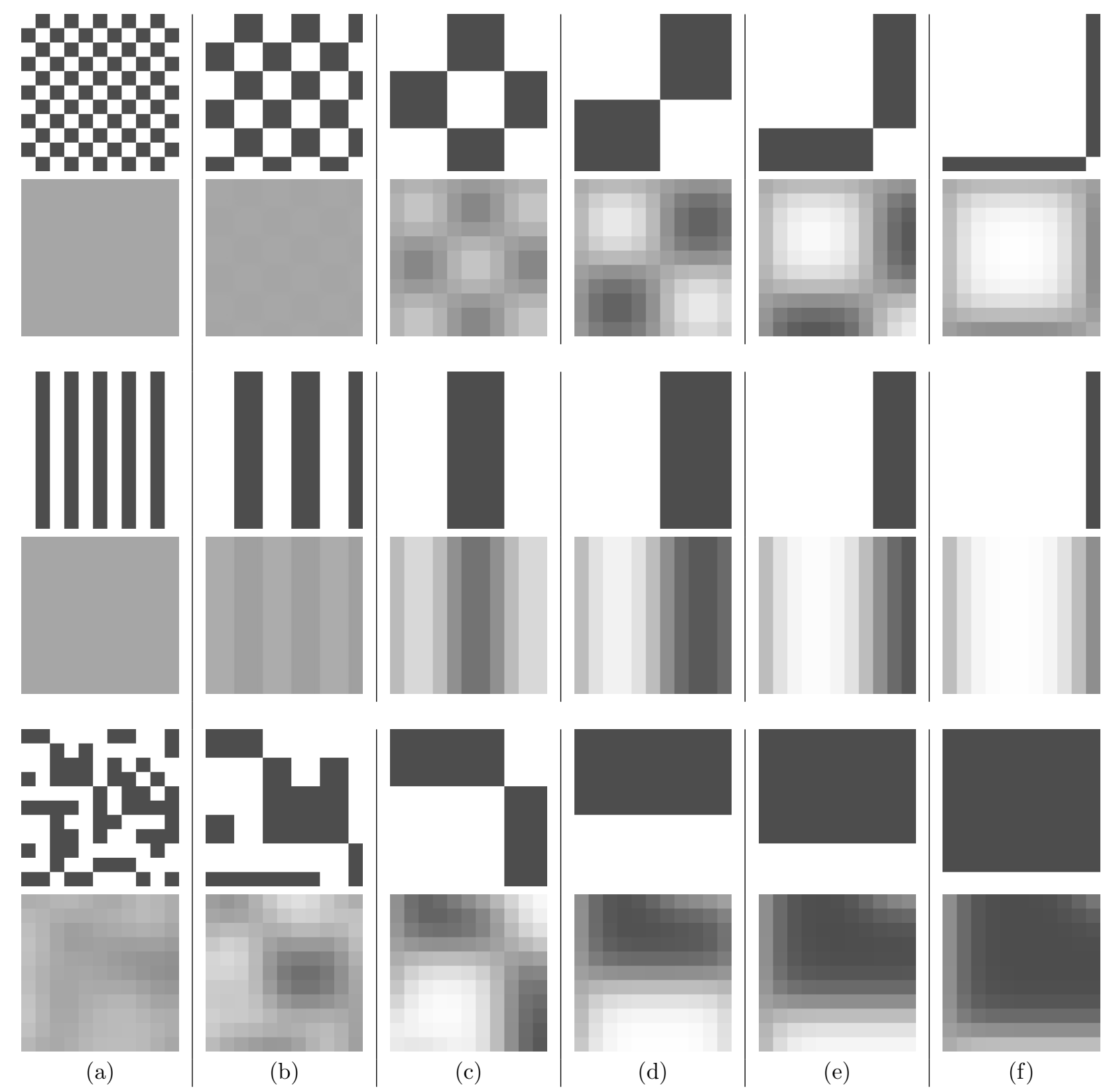

Figure 7 . View of a $11 \times 11$ processed patch with and without blur respectively, with a checkerboard pattern (top row), a line pattern (middle row) and a binary random 50/50 pattern (bottom row). For each pattern, five structural element sizes are displayed : (a) $s=1$, (b) $s=2$, (c) $s=4$, (d) $s=6$, (e) $s=8$, (f) $s=10$. 


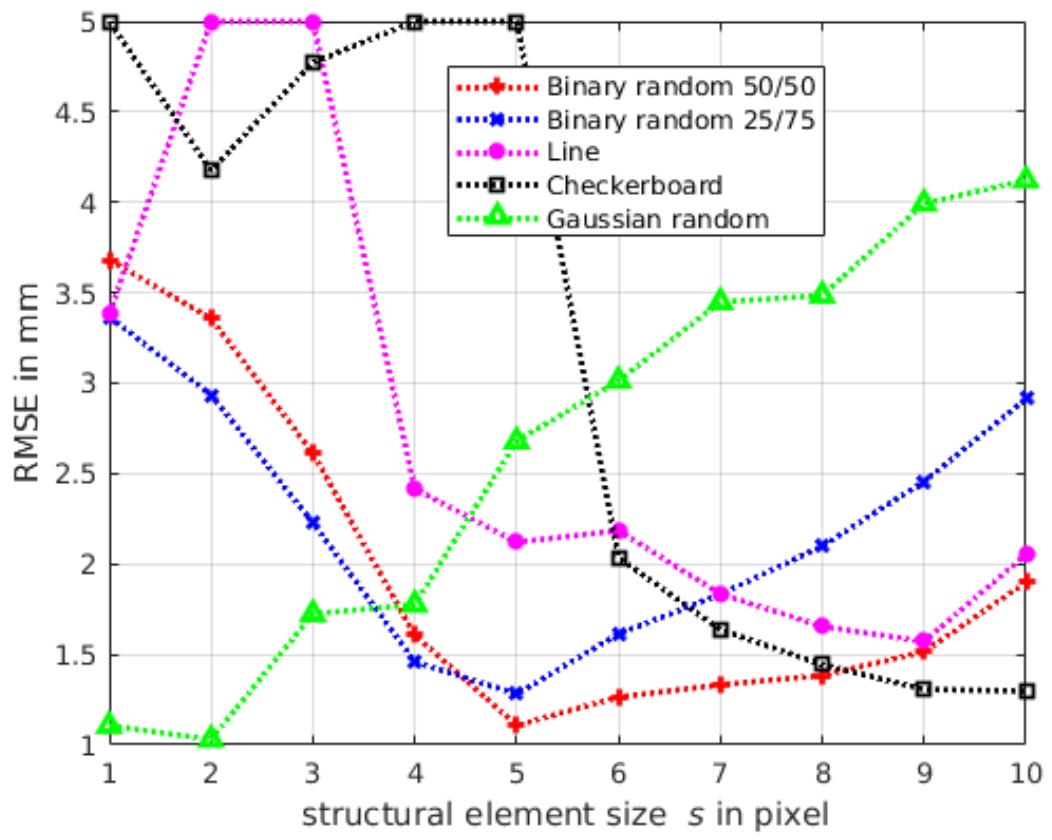

Figure 8. Average Root Mean Square Error of depth estimation calculated on simulated images, with respect to the scale factor $s$ and various projected patterns.

RMSE are truncated when it is higher than $5 \mathrm{~mm}$. For comparative purpose, we also compute depth estimation for each simulated blurred metal scene without projected pattern: the average RMSE is then $9 \mathrm{~mm}$.

Figure 7 shows a $11 \times 11$ pixel patch for several structural elements sizes with and without defocus blur (corresponding to a depth of $310 \mathrm{~mm}$ ).

Several conclusions can be drawn from these simulations. First, all projected patterns improve depth estimation with respect to the case of passive DFD (without projection). Then, except for the Gaussian pattern, with too small $(s<4$ pixels) or too large $(s>10$ pixels) structural elements, depth estimation shows poor results. This can be explained when looking at Figure 7. Indeed, for a projected pattern having a small structural element and an 50/50 distribution of black and white elements, the image patch becomes almost homogeneous when blur is significant. Hence, depth estimation can not be efficient. On the other hand, for large structural elements $(s>10)$, the patch is completely filled with one structural element, the scene itself is then homogeneous which is also not efficient for DFD. The best performances are obtained for values of $s$ around $5-6$. We can observe that its corresponds to a structural element approximately half as large as the patch size. For the Gaussian pattern, larger is the structural element more important is the decrease of the contrast in the patch, resulting in a worst estimation when $s$ increases.

To conclude, best simulation results are obtained with the binary 50/50 random pattern for a structural element size of half as large as the patch size.

\section{EXPERIMENTAL EVALUATION}

\subsection{Practical constraints}

Several practical constraints appear when adapting the simulation evaluation to the experimental one. First, the projected structural element is affected by projector resolution which induces a residual blur on the projected scene which is not due to defocus. This effect is more important as the structural element size is small. Another concern is related to the variation of the distance between the projector and the object plane, which entails a slight variation of the scale in the projected pattern (telecentric projection used by Nayar ${ }^{8}$ can solve this 
difficulty). In our experiments, both problems are avoided, as structural elements used are large enough not to be degraded by projector resolution. Moreover the experimental range used is short enough making the scale variation of the projection negligible. Regarding the image, we have to take into account that PSFs vary off-axis because of various optical aberrations. Since calibration of the camera is simply made on-axis (in the center of the image), depth quantitative evaluation is also performed on-axis, in a square window of $121 \times 121$ pixels, and we expect the depth map quality to decrease away from the image center.

\subsection{Quantitative evaluation}

In this section, we present quantitative experimental evaluation of depth estimation accuracy on real frontoparallel plane illuminated by projected pattern. The description of the global system is given in Section 4.1. A planar surface is put on the motion controller and a sheet of paper is pasted to it. A translation is carried out by the controller between 310 and $340 \mathrm{~mm}$ of the camera while we assure that the plane remains fronto-parallel to the sensor. Projector is placed $80 \mathrm{~cm}$ from the scene and is focused in the middle of the working range. In this configuration the whole working range is contained within the projector depth of field. As binary random 50/50 pattern and the Gaussian pattern have shown the best results in simulation, we run experimental evaluations for these two textures. Depth maps obtained with the proposed DFD algorithm and a patch size of $11 \times 11$ pixels are cropped in a centered window of $121 \times 121$ pixels. The size of the projected pattern is chosen in order to have the structural element being half the size of the estimation patch. In Figure 9 are displayed experimental captures of respectively : a binary random 50/50 pattern window and patch in (a) and (b) and Gaussian texture window and patch in (c) and (d), at depth $310 \mathrm{~mm}$. This figure illustrates that the processed patches are similar to the simulated ones in terms of pattern scale.

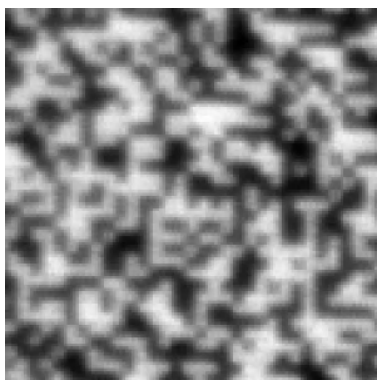

(a)

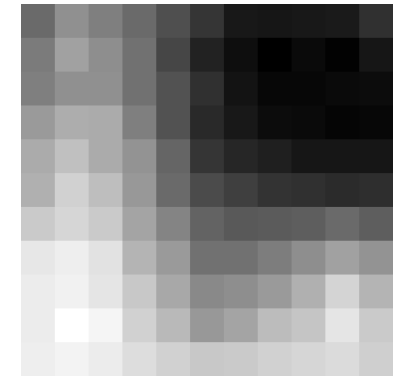

(b)

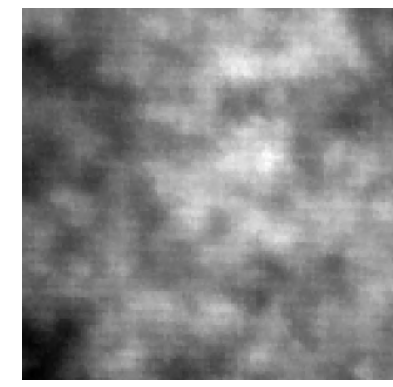

(c)

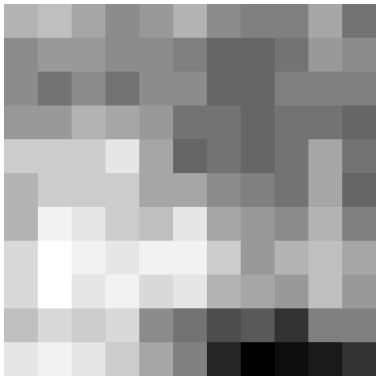

(d)

Figure 9. Experimental view of the depth estimation evaluation window $(121 \times 121$ pixels $)$ and of a processed patch $(11 \times 11$ pixels in the middle of the window), respectively (a) and (b) for a binary random 50/50 pattern, (c) and (d) for a Gaussian texture.

Figure 10 shows the mean and the standard deviation of depth estimated by the DFD algorithm inside the centered evaluation window at each depth for each of the two patterns. We remove $5 \%$ of the extreme depth estimation values in order to remove outliers in the statistics. RMSE of the depth estimation relative to the true depth is also computed. For the binary pattern, the RMSE is of $0.96 \mathrm{~mm}$ and standard deviation is of 1.5 $\mathrm{mm}$. For the Gaussian pattern, the RMSE is of $1.8 \mathrm{~mm}$ and the mean standard deviation is of $2.4 \mathrm{~mm}$. This experiment confirms that the binary random 50/50 pattern gives the best results (lowest bias and variance with the respect to the ground truth) which is consistent with simulations performed in Section 5.

\subsection{Qualitative evaluation}

The proposed method has also been used on real industrial object. Figure 11 shows depth maps obtained with our active chromatic DFD method using a $11 \times 11$ pixel square patch and the corresponding map of estimated $\widehat{\alpha}$, see (13)), $\alpha$ that can be seen as an inverse signal to noise ratio estimation (SNR). The top line shows results while only a white light without pattern is projected on the object. The bottom line shows results with the binary random pattern. A $3 \times 3$ median filter is applied to regularize the depth map. Black label corresponds to outlier regions characterized by $\widehat{\alpha}>1$. 


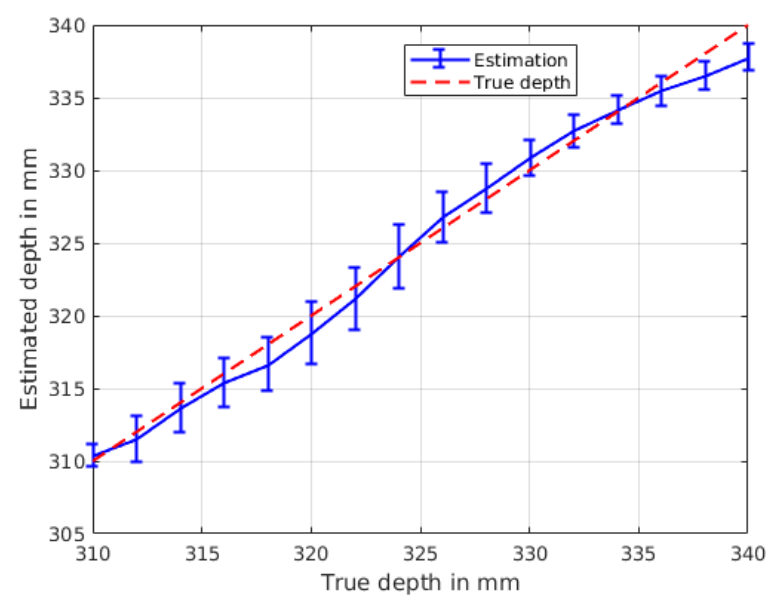

(a)

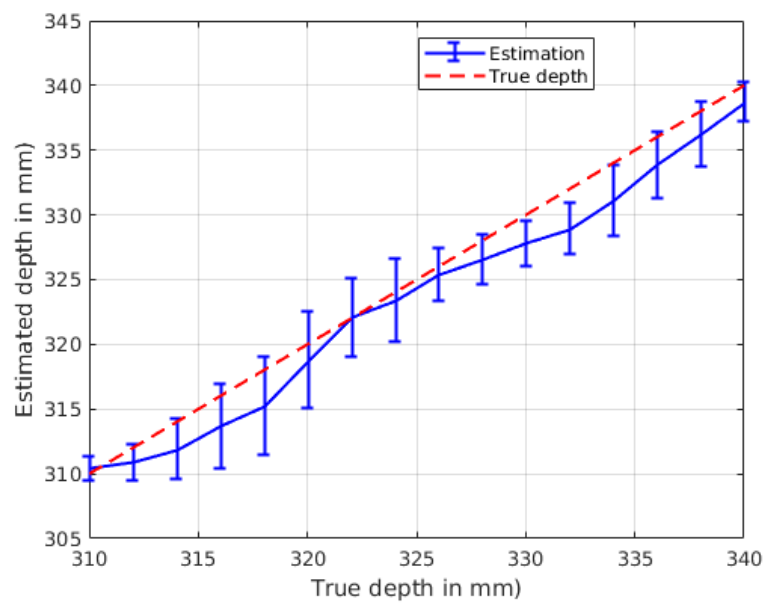

(b)

Figure 10. Depth estimation performance of two projected patterns on a fronto-parallel plane between 310 and 340 mm of the camera. (a) Binary random 50/50 pattern, (b) Gaussian pattern.

Without the pattern projection, the image shows homogeneous regions (mostly in the right-bottom of the piece) and light reflections (mostly in the top part of the piece). Hence, some outlier zones in black and wrongly estimated zones appear in the depth map. On the other hand, with the projected pattern, the image shows no homogeneous region and less reflections, which induce a reduced number of outliers in the depth map as well as a reduction of estimated $\alpha$ (in other words the SNR is improved). Regarding the accuracy of depth estimation, the depth map obtained with the projected pattern one is the closest to ground truth, which is built manually using calipers and shown in Figure 12. Note, however that as PSFs were calibrated on-axis of the chromatic lens, estimation is more relevant in the centered region of the image. An off axis calibration of the PSF could be conducted to improve the off-axis results. ${ }^{6}$

\section{CONCLUSION AND PERSPECTIVES}

In this paper we have proposed a new concept for compact 3D sensor dedicated to industrial inspection, combining chromatic Depth from Defocus (DFD) and structured illumination. In contrast with state of the art active DFD using sparse point or line pattern that lead to sparse depth maps, we have optimized an original dense texture to project to the scene in order to produce dense depth maps. We have shown that a binary random noise pattern gives the best performances, with a structural element half the size of the processed patch. Finally we have presented preliminary results of active chromatic DFD on an industrial metal object.

There are several perspectives for this work. First, in this paper, we have used an algorithm originally dedicated to DFD with natural scenes, based on a Gaussian prior. The objective is now to modify the scene prior to be consistent with the projected pattern. Potential experimental issues of active DFD such as light reflection and blur due to projector resolution have been mitigated in our experiments. Future works will also focus on improving the experimental settings, using for instance polarization filters ${ }^{20}$ or specific algorithm ${ }^{21}$ to completely overcome reflections. Finally, our active 3D system is materially limited by the projector (resolution and working range), and by the characteristics of the chromatic lens (variation of chromatic blur and off-axis aberrations). Using the co-design approach presented by Trouve et al. ${ }^{22}$ the design of the global system could be optimized to improve the overall performance of the 3D sensor. 

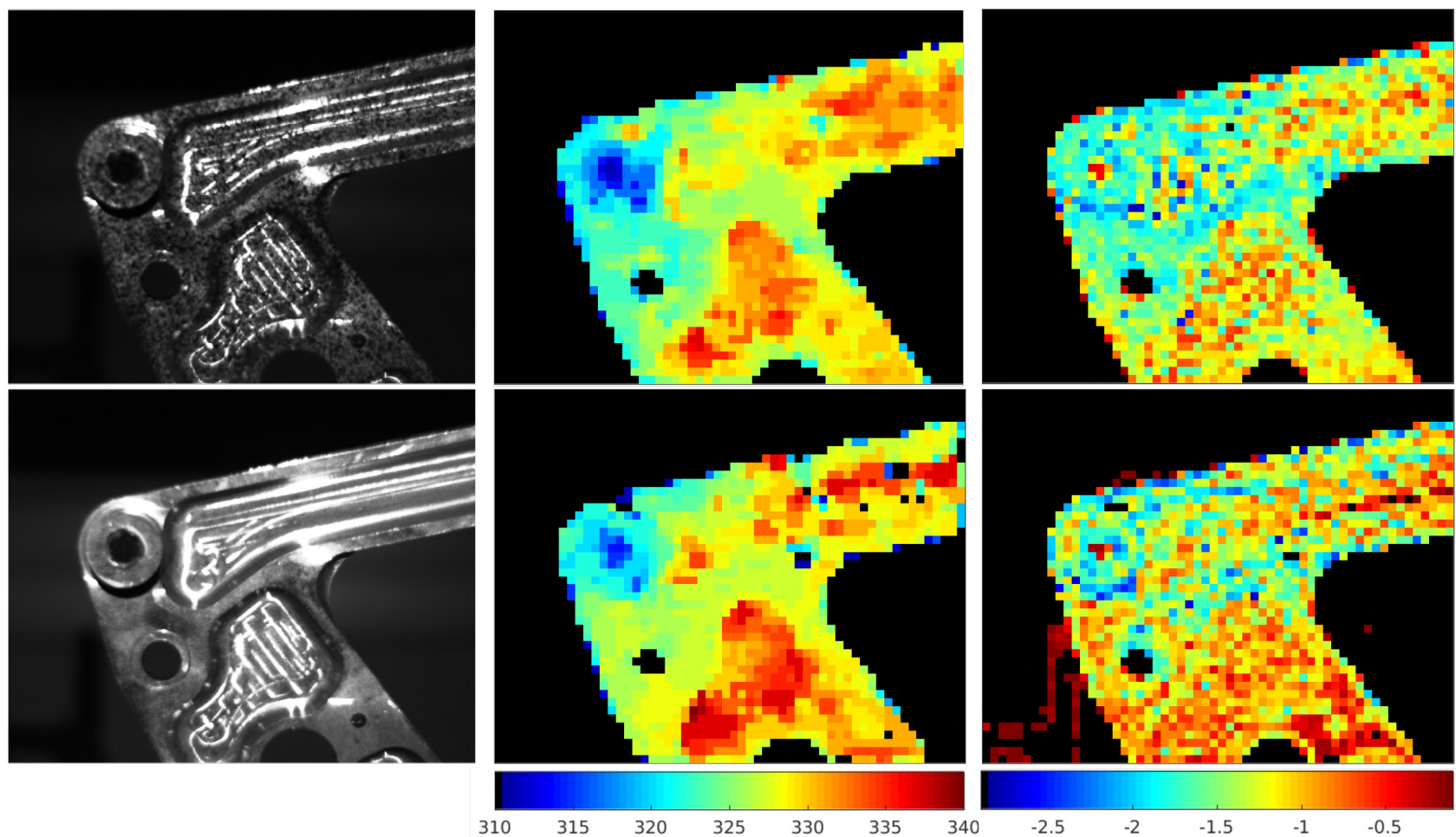

Figure 11. Results of depth map estimation of a metal object without projected pattern (top) and with binary random pattern (bottom). From left to right: acquired image, depth map, $\log (\alpha)$ map. The depth labels are in mm. Black label corresponds to homogeneous regions characterized by $\widehat{\alpha}>1$.

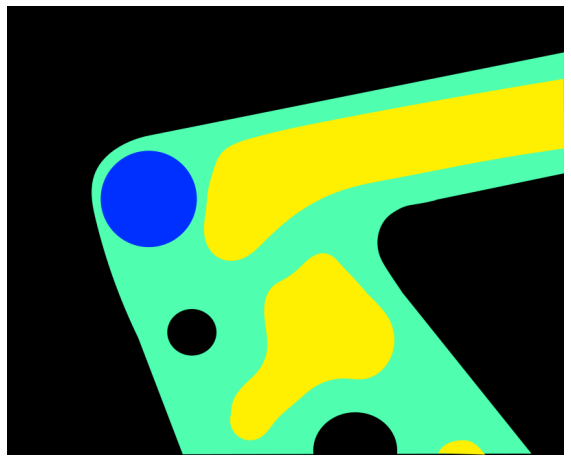

Figure 12. Ground truth of the metal industrial piece built manually using calipers. 


\section{REFERENCES}

[1] Savio, E., Chiffre, L. D., and Schmitt, R., "Metrology of freeform shaped parts," CIRP Annals 56(2), 810 - 835 (2007).

[2] Pentland, A. P., "A new sense for depth of field," IEEE Transactions on Pattern Analysis and Machine Intelligence (July 1987).

[3] Grossmann, P., "Depth from focus," Pattern Recognition Letters 5(1), 63 - 69 (1987).

[4] Levin, A., Fergus, R., Durand, F., and Freeman, W. T., "Image and depth from a conventional camera with a coded aperture," in [ACM SIGGRAPH 2007 Papers], SIGGRAPH '0\%, ACM, New York, NY, USA (2007).

[5] Chakrabarti, A. and Zickler, T., "Depth and deblurring from a spectrally varying depth of field," in [European Conference on Computer Vision], European Conference on Computer Vision (2012).

[6] Trouvé, P., Champagnat, F., Besnerais, G. L., Sabater, J., Avignon, T., and Idier, J., "Passive depth estimation using chromatic aberration and a depth from defocus approach," Appl. Opt. 52, 7152-7164 (Oct 2013).

[7] Ng, R., Levoy, M., Brédif, M., Duval, G., Horowitz, M., Hanrahan, P., et al., "Light field photography with a hand-held plenoptic camera," Computer Science Technical Report CSTR 2(11), 1-11 (2005).

[8] Nayar, S. K., Watanabe, M., and Noguchi, M., "Real-time focus range sensor," IEEE Transactions on Pattern Analysis and Machine Intelligence 18, 1186-1198 (Dec 1996).

[9] Ghita, O., Whelan, P. F., and Mallon, J., "Computational approach for depth from defocus," Journal of Electronic Imaging 14, $14-14-8$ (2005).

[10] Zhang, L. and Nayar, S., "Projection defocus analysis for scene capture and image display," ACM Trans. Graph. 25, 907-915 (July 2006).

[11] Lertrusdachakul, I., Fougerolle, Y., and Laligant, O., "Dynamic (de)focused projection for three-dimensional reconstruction," Optical Engineering 50, 113201 (Oct. 2011).

[12] Girod, B. and Scherock, S., "Depth from defocus of structured light," Proc.SPIE 1194, $1194-1194-7$ (1990).

[13] Moreno-Noguer, F., Belhumeur, P. N., and Nayar, S. K., "Active refocusing of images and videos," $A C M$ Transactions On Graphics (TOG) 26(3), 67-es (2007).

[14] Masuyama, H., Kawasaki, H., and Furukawa, R., "Depth from projector's defocus based on multiple focus pattern projection," IPSJ Transactions on Computer Vision and Applications 6, 88-92 (2014).

[15] Ma, B., Computational Depth from Defocus via Active Quasi-random Pattern Projections, Master's thesis (2018).

[16] Trouvé, P., Champagnat, F., Besnerais, G. L., and Idier, J., "Single image local blur identification," in [2011 18th IEEE International Conference on Image Processing], 613-616 (Sept 2011).

[17] Condat, L., "A generic variational approach for demosaicking from an arbitrary color filter array," IEEE (2009).

[18] Reichenbach, S. E., Park, S. K., and Narayanswamy, R., "Characterizing digital image acquisition devices," Optical Engineering 30(2), 170-178 (1991).

[19] Kwitt, R. and Meerwald, P., "Salzburg texture image database." http://www.wavelab.at/sources/STex/ (2013).

[20] Wolff, L. B. and Boult, T. E., "Constraining object features using a polarization reflectance model," PhysicsBased Vision: Principles and Practice: Radiometry 1, 167 (1993).

[21] Sun, X., Liu, Y., Yu, X., Wu, H., and Zhang, N., "Three-dimensional measurement for specular reflection surface based on reflection component separation and priority region filling theory," Sensors 17(1), 215 (2017).

[22] Trouvé, P., Champagnat, F., Le Besnerais, G., Druart, G., and Idier, J., "Design of a chromatic 3d camera with an end-to-end performance model approach," in [The IEEE Conference on Computer Vision and Pattern Recognition (CVPR) Workshops], (June 2013). 